\title{
Schwannoma of the medial cutaneous nerve of the arm: a rare location with concomitant compression neuropathy of the ulnar nerve
}

\author{
Jerzy Gosk ${ }^{1}$, Olga Gutkowska ${ }^{1}$, Jacek Martynkiewicz ${ }^{1}$, Michał Bąk ${ }^{1}$, Agnieszka Hałoń ${ }^{2}$ \\ ${ }^{1}$ Department of Traumatology, Clinic of Traumatology and Hand Surgery, Wroclaw Medical University \\ ${ }^{2}$ Division of Pathomorphology and Oncological Cytology, Wroclaw Medical University
}

\begin{abstract}
The objective of this paper is to present a case of rare location of schwannoma in the medial cutaneous nerve of the arm at the level of the axilla. Preliminary diagnostic examination of the tumour was carried out in another hospital by means of open biopsy. In the preoperative period symptoms of ulnar nerve dysfunction (paraesthesias, positive Hoffmann-Tinel sign) dominated the clinical picture. After having performed imaging studies and electromyographic $(E M G)$ examination, the patient was scheduled for an operation. The tumour, measuring $3.5 \times 3.0 \times 1.5 \mathrm{~cm}$, was resected without damage to the fascicular structure. Presence of paraesthesias in the distribution of the medial cutaneous nerve of the arm, which was first noted in the postoperative period, persists in moderate severity until now. As a result of the performed operative treatment, such symptoms as palpable tumour mass, pain, paraesthesias in the ulnar nerve distribution and positive Hoffmann-Tinel sign resolved. On the basis of histopathological examination results the final diagnosis of classical schwannoma was established.
\end{abstract}

Key words: schwannoma, nerve compression, tumour, medial cutaneous nerve of the arm.

\section{Introduction}

The medial cutaneous nerve of the arm is one of the smallest branches of the brachial plexus [20]. It is a nerve arising from the medial cord (C8, Th1) $[16,20]$. The medial cutaneous nerve of the arm passes through the axilla, crossing posterior to the axillary vein and lying medially to it. In its further course it is located medially to the brachial artery and the basilic vein $[16,20]$. At the mid-length of the arm the nerve pierces the deep fascia and runs toward the skin $[16,20]$. The medial cutaneous nerve of the arm supplies the skin of the medial aspect of the arm (the anterior aspect of the distal third of the arm and most of the posteromedial aspect) [16]. This nerve can communicate with the intercostobrachial nerve and medial cutaneous nerve of the forearm [2].

The medial cutaneous nerve of the arm can be subjected to damage due to trauma (including that of iatrogenic aetiology), inflammatory factors or as a result of development of neoplastic lesions affecting the nerve or adjacent structures [9]. 


\section{Case report}

A 51-year-old woman was admitted to our unit for surgical treatment of a tumour located in her left axillary fossa. The tumour was first noticed by the patient about 6 months earlier. There was no history
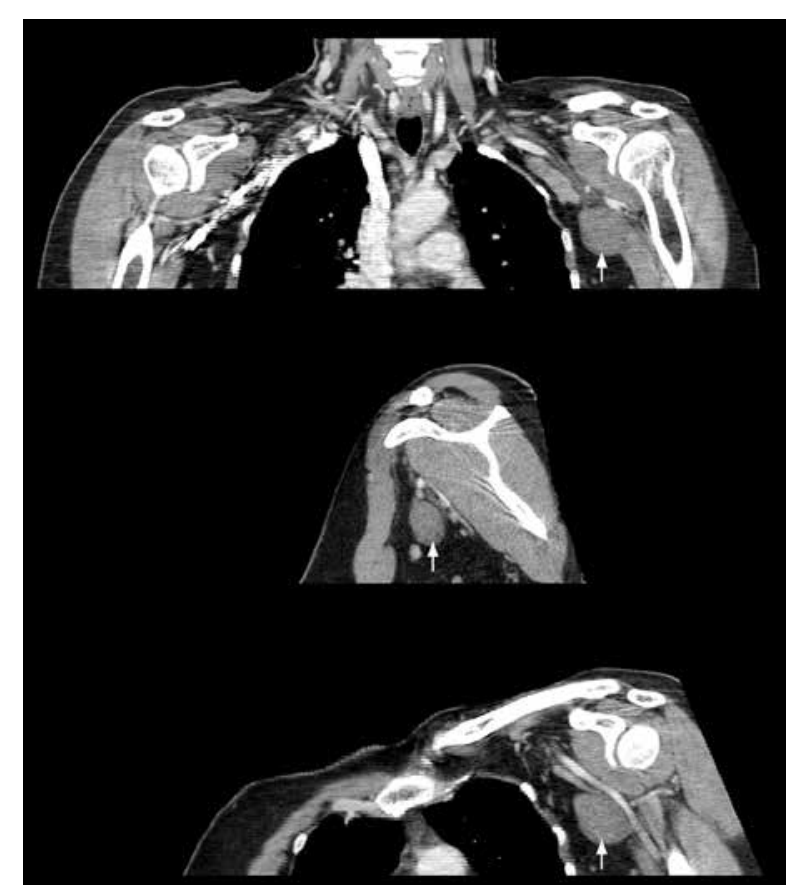

Fig. 1. Computed tomography scans of the chest and left shoulder showing the tumour (white arrows) located in the left axillary fossa. of trauma or inflammation of the affected area. On anamnesis, the patient reported pain, aggravated by applying pressure to the tumour mass and by overstraining the upper limb, and presence of paraesthesias in the $4^{\text {th }}$ and $5^{\text {th }}$ fingers of her left hand. Before admission to our clinic, the patient had an open biopsy of the tumour performed in another hospital (27.03.2013). On the basis of histopathological analysis of the biopsied material, the diagnosis of schwannoma was established. In the computed tomography (CT) scan performed before the biopsy (08.07.2013), presence of a smoothly contoured solid tumour measuring $3.2 \times 2.8 \times 3.6 \mathrm{~cm}$ was detected. The tumour was hypo/isodense (20-40 H), with no significant contrast enhancement. Medially and anteriorly it was difficult to determine the boundaries between the tumour and the short head of the biceps brachii muscle and coracobrachialis muscle (Fig. 1). On magnetic resonance imaging (MRI) examination performed after the biopsy (29.08.2014), presence of a tumour measuring $3.7 \times 2.3 \times 3.5 \mathrm{~cm}$ was demonstrated. The tumour had clear outer boundaries and did not compress the blood vessels of the axillary fossa. After administration of contrast agent, homogeneous signal enhancement was noted (Fig. 2).

On EMG examination performed on 29.08.2013 no characteristics of significant function impairment of the median, ulnar and radial nerves were detect-
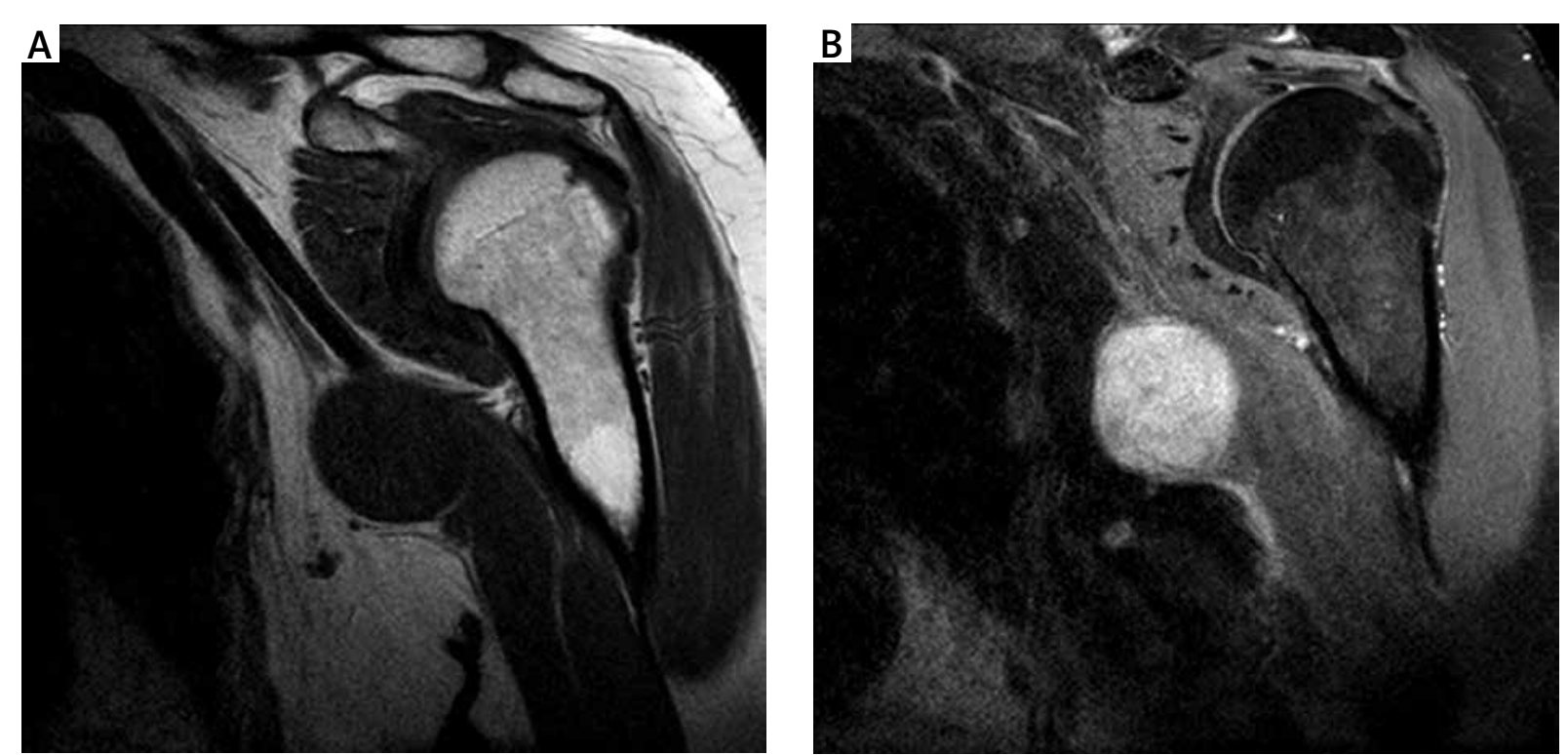

Fig. 2. Magnetic resonance imaging scans demonstrating: A) the tumour location in the left axillary fossa;

B) signal enhancement of the lesion after administration of contrast agent. 

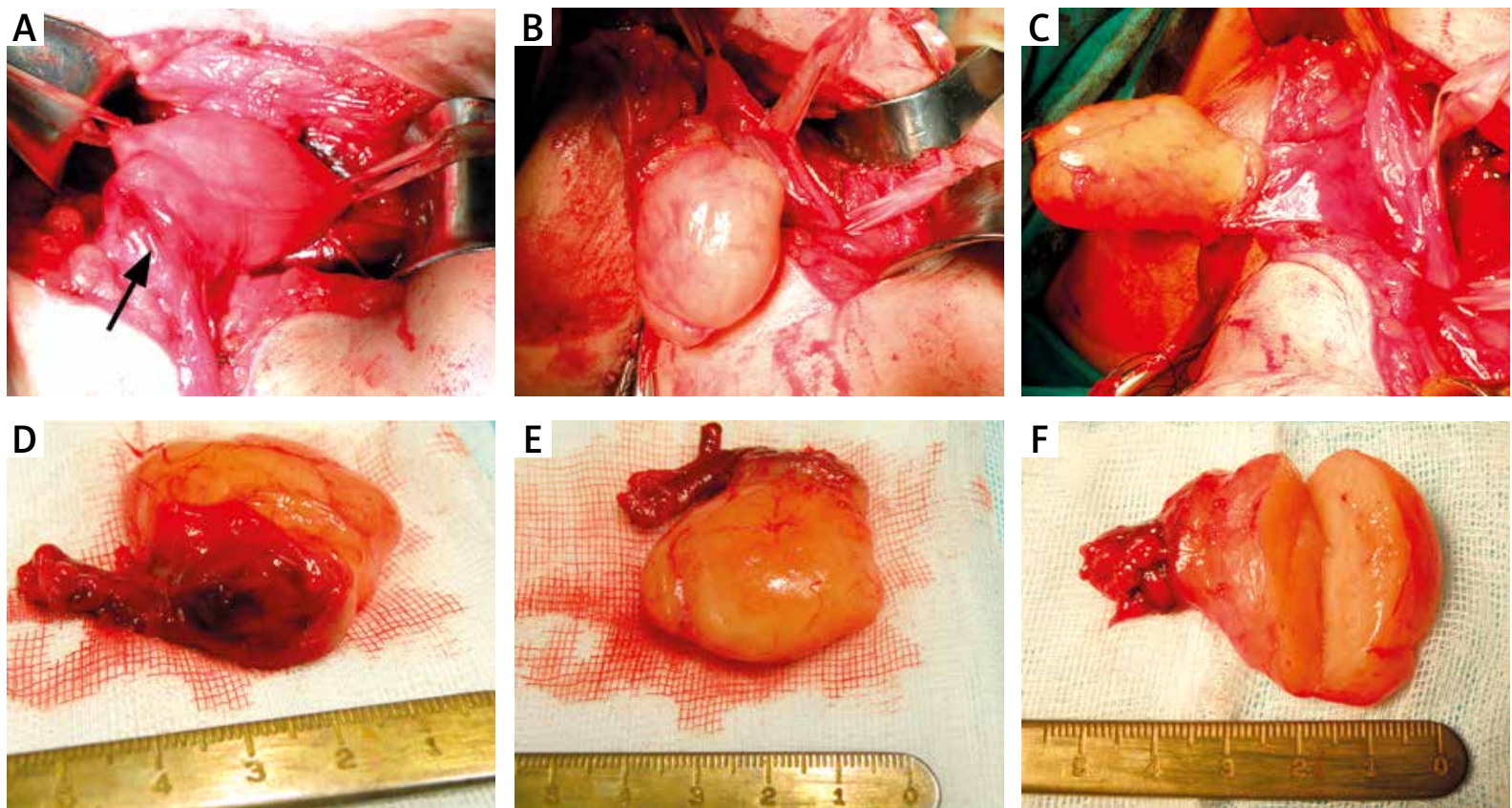

Fig. 3. Removal of the tumour from the medial cutaneous nerve of the arm: A) intraoperative view: exposure of the tumour (the site of previously performed biopsy is visible - black arrow); B, C) intraoperative view: status after dissection of the tumour; D, E) postoperative view: appearance of the tumour after resection; F) cross-section of the tumour.

ed. The medial cutaneous nerve of the arm was not subjected to assessment.

On clinical examination a palpable tumour mass was present in the left axillary fossa. Pain was triggered by applying pressure to the tumour. On percussion of the tumour, a positive Hoffmann-Tinel sign was elicited, with the presence of paraesthesias in the $4^{\text {th }}$ and $5^{\text {th }}$ fingers of the patient's left hand. Superficial sensory function examination (static and dynamic sensory discrimination) of this area did not reveal significant impairment in comparison to the opposite side. No muscle atrophy was observed in the patient's left upper limb.

The patient was scheduled for an operation. Surgery was performed on 18.11.2013. After exposure of the tumour mass and identification of the adjacent neural structures, it was determined that the tumour originated from the medial cutaneous nerve of the arm. The tumour, measuring $3.5 \times 3.0 \times 1.5 \mathrm{~cm}$, was dissected from the nerve without damage to the fascicular structure. The operation was performed with the use of microsurgical instruments and an operating microscope. Marked hardening and scarring of tissues at the site of previously performed open biopsy significantly hindered the dissection and enucleation of the tumour (Fig. 3).

Classical schwannoma was diagnosed on the basis of postoperative histopathological examination results - no. 33695 from 03.12.2013 (Fig. 4). In the early postoperative period, onset of paraesthesias in the distribution of the medial cutaneous nerve of the arm was observed. During the follow-up period of one year, no symptoms of tumour recurrence were detected, and the pain and paraesthesias in the ulnar nerve distribution resolved. Hoffmann-Tinel sign is negative. Paraesthesias of moderate severity in the distribution of the medial cutaneous nerve of the arm still persist.

\section{Discussion}

The case of schwannoma located in the medial cutaneous nerve of the arm presented in this paper is interesting for a number of reasons. Firstly, this location of schwannoma is extremely rare in clinical practice. In the upper limb, these tumours are most commonly located in the ulnar, median and radial nerves, being significantly rarer in the musculocutaneous and axillary nerves $[1,3,5,6,18]$. In our 

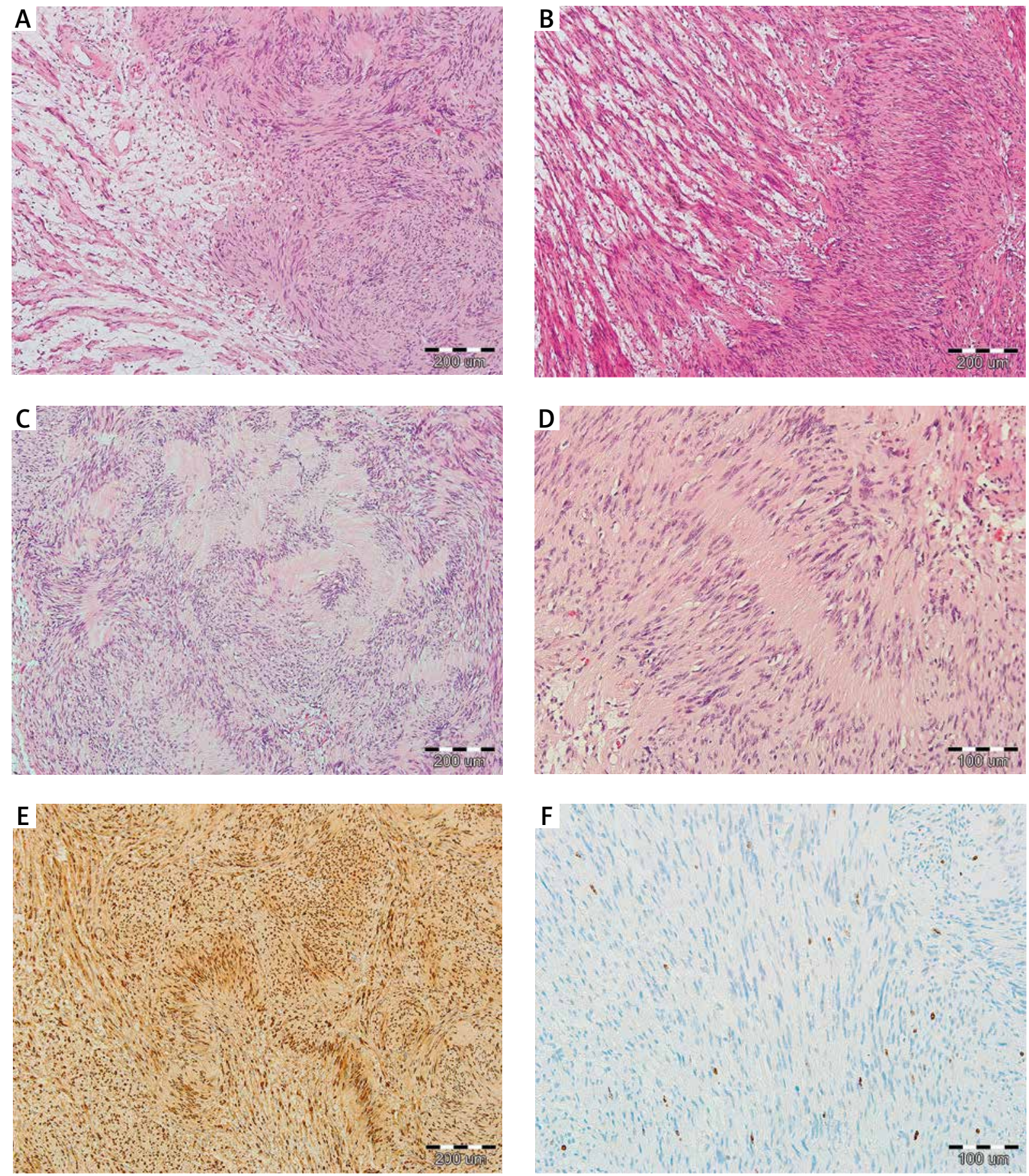

Fig. 4. Histological features of classical schwannoma: A) two typical histological patterns: Antoni A pattern Verocay bodies as cellular areas surrounded by nuclear palisades (right side of the image) opposed to less cellular Antoni B pattern (left side of the image); B) cellular Antoni A area (right side of the image) and loose paucicellular Antoni B area with sharp border between them; C) typical Verocay bodies (Antoni A pattern); D) prominent nuclear palisading; E) diffuse positive immunostaining for S-100 protein; F) proliferative index Ki-67 positive, low (1\%). 
material comprising 101 cases of peripheral nerve tumours treated operatively between the years 1983 and 2012, we observed only one case of schwannoma located in the medial cutaneous nerve of the arm in a 24-year-old male patient [6]. We have not found a single case report describing a schwannoma in this location in the available literature. Secondly, symptoms observed in the preoperative period suggested location of the tumour in the ulnar nerve. Positive Hoffmann-Tinel sign and presence of paraesthesias in the $4^{\text {th }}$ and $5^{\text {th }}$ fingers of the patient's left hand were detected on clinical examination. The above symptoms resulted from compression of the adjacent ulnar nerve, also originating from the medial cord of the brachial plexus, by the tumour mass. At the same time, less evident symptoms of dysfunction of the nerve being the site of tumour origin can result from the less important role of the medial cutaneous nerve of the arm in innervation of the upper limb. The medial cutaneous nerve of the arm is purely sensory. Impairment of its function does not affect the functionality of the upper limb. For this reason, dysfunction of the medial cutaneous nerve of the arm does not cause evident discomfort in patients, in contrast to impairment of function of other nerves $[2,16,20]$. Detection of its potential pathologies is also difficult due to the fact that this nerve is rarely subjected to assessment during electrodiagnostic testing [9].

Thirdly, difficulties in tumour dissection resulting from previously performed open biopsy of the tumour made us wonder whether it is necessary and useful to perform a biopsy of a peripheral nerve tumour preoperatively. Tumour biopsy can be performed as percutaneous fine-needle or core needle biopsy or as an open biopsy [17]. Closed biopsy techniques of a soft tissue mass are highly diagnostic, with a low complication rate $[13,21]$. However, biopsy of a nerve tumour is connected with the risk of certain complications. The possibility of haemorrhage and damage to viable fascicles has been highlighted $[3,11,18]$. It may lead to secondary intraneural scarring and aggravation of neuropathic pain $[3,10,11]$. Some authors emphasise increased risk of development of new, postoperative neurological deficits in patients who underwent a previous invasive procedure at the surgical site $[4,10,12,15]$. Levi et al. observed new, postoperative neurological deficits in $41 \%$ of their patients who had previously been subjected to such procedures (biopsy or oper- ation) at the surgical site [12]. It is also important to be aware that the results obtained after biopsy are not always identical to the results of histopathological examination of the surgically removed tumour [1,17-19]. It has been suggested that biopsy is recommended in case of a suspected malignant lesion $[3,14]$. At the same time, the possibility of spread of malignant neoplastic cells as a result of tumour biopsy has been emphasised $[7,18]$.

In the above-described case, the patient underwent an open tumour biopsy in another hospital before definitive operative treatment. Histopathological examination of the removed sample revealed a schwannoma. This result, obtained after the first operation, neither changed further management nor made it possible to determine the site of tumour origin.

In order to remove the entire tumour, another operation was required, which was performed in conditions altered by the previous surgery and therefore technically more demanding. In our opinion, incisional biopsy is only recommended in cases in which presence of a large tumour with focal necrosis, haemorrhage and infiltration of surrounding tissues is observed intraoperatively. This is compatible with observations made by other authors $[7,8]$. In the above-described case an increase in intensity of paraesthesias in the distribution of the medial cutaneous nerve of the arm was seen in the postoperative period. This observation may to some extent confirm suggestions regarding the risk connected with performing preoperative tumour biopsy.

\section{Conclusions}

A case of very rare location of classical schwannoma in the medial cutaneous nerve of the arm with concomitant symptoms of ulnar nerve irritation has been presented in this paper. Preoperative open biopsy of the tumour constituted grounds for questioning the appropriateness and the risk connected with this type of management of peripheral nerve tumours.

\section{Acknowledgements}

We would like to thank Bartosz Witkowski for providing medical writing service on behalf of Wroclaw Medical University.

\section{Disclosure}

Authors report no conflict of interest. 


\section{References}

1. Adani R, Baccarani A, Guidi E, Tarallo L. Schwannomas of the upper extremity: diagnosis and treatment. Chir Organi Mov 2008; 92: 85-88.

2. Blanco R, Jiménez Gómez BM, López González JM. Ultrasound Appearance of the Cutaneous Nerves of the Upper Limb: A Novel Description in Pain Management. J Pain Relief 2012; 1: 2.

3. Date R, Muramatsu K, Ihara K, Taguchi T. Advantages of intracapsular micro-enucleation of schwannoma arising from extremities. Acta Neurochir (Wien) 2012; 154: 173-178; discussion 178.

4. Donner TR, Voorhies RM, Kline DG. Neural sheath tumors of major nerves. J Neurosurg 1994; 81: 362-373.

5. El Andaloussi Y, Abkari I, Bleton R. Schwannome du nerf axillaire (à propos d'un cas) [Axillary nerve schwannoma (case report)]. Chir Main 2008; 27: 232-234.

6. Gosk J, Rutowski R, Rabczyński J. Peripheral nerve tumours in own material. Folia Neuropathol 2004; 42: 203-207.

7. Gupta G, Mammis A, Maniker A. Malignant peripheral nerve sheath tumors. Neurosurg Clin N Am 2008; 19: 533-543.

8. July J, Guha A. Treatment of the peripheral nerve tumors. CDK 185 2011; 38: 257-262.

9. Jung MJ, Byun HY, Lee CH, Moon SW, Oh MK, Shin H. Medial antebrachial cutaneous nerve injury after brachial plexus block: two case reports. Ann Rehabil Med 2013; 37: 913-918.

10. Knight DM, Birch R, Pringle J. Benign solitary schwannomas: a review of 234 cases. J Bone Joint Surg Br 2007; 89: 382-387.

11. Kubiena $H$, Entner T, Schmidt M, Frey M. Peripheral neural sheath tumors (PNST) - what a radiologist should know. Eur J Radiol 2013; 82: 51-55.

12. Levi AD, Ross AL, Cuartas E, Qadir R, Temple HT. The surgical management of symptomatic peripheral nerve sheath tumors. Neurosurg 2010; 66: 833-840.

13. Malizos K, Ioannou M, Kontogeorgakos V. Ancient schwannoma involving the median nerve: a case report and review of the literature. Strat Trauma Limb Recon 2013; 8: 63-66.

14. Matejcik V. Our experience with surgical treatment of the schwannomas of peripheral nerves. Bratisl Lek Listy 2002; 103 477-479.

15. Miles S-J, Amirfeyz R, Bhatia R, Leslie I. Benign soft tissue tumours of the hand. Orthop Trauma 2010; 24: 181-185.

16. Race CM, Saldana MJ. Anatomic course of the medial cutaneous nerves of the arm. J Hand Surg Am 1991; 16: 48-52.

17. Resnick JM, Fanning CV, Caraway NP, Varma DG, Johnson M. Percutaneous needle biopsy diagnosis of benign neurogenic neoplasms. Diagn Cytopathol 1997; 16: 17-25.

18. Siqueira MG, Socolovsky M, Martins RS, Robla-Costales J, Di Masi G, Heise CO, Cosamalón JG. Surgical treatment of typical peripheral schwannomas: the risk of new postoperative deficits. Acta Neurochir (Wien) 2013; 155: 1745-1749.

19. Sohn YM, Kim SY, Kim EK. Sonographic appearance of a schwannoma mimicking an axillary lymphadenopathy. J Clin Ultrasound 2011; 39: 477-479.

20. Tubbs RS, Jones VL, Loukas M, Cömert A, Shoja MM, Wellons JC 3rd, Cohen-Gadol AA. Anatomy and landmarks for branches of the brachial plexus: a vade mecum. Surg Radiol Anat 2010; 32: 261-270.
21. Welker JA, Henshaw RM, Jelinek J, Shmookler BM, Malawer MM. The percutaneous needle biopsy is safe and recommended in the diagnosis of musculoskeletal masses. Cancer 2000; 89: 2677-2686. 